CAHIERS DE

NARRATOLOGIE

\section{Cahiers de Narratologie}

Analyse et théorie narratives

37 | 2020

Approches transmédiales du récit dans les fictions contemporaines

\title{
Écosystème médiatique et circulation de la fiction : Dragon Ball, Pokémon, Sword Art Online
}

\section{Bounthavy Suvilay}

\section{(2) OpenEdition}

\section{Journals}

Édition électronique

URL : http://journals.openedition.org/narratologie/10613

DOI : 10.4000/narratologie.10613

ISSN : 1765-307X

Éditeur

LIRCES

Référence électronique

Bounthavy Suvilay, «Écosystème médiatique et circulation de la fiction : Dragon Ball, Pokémon, Sword Art Online », Cahiers de Narratologie [En ligne], 37 | 2020, mis en ligne le 04 septembre 2020, consulté le 06 septembre 2020. URL : http://journals.openedition.org/narratologie/10613 ; DOI : https://doi.org/ 10.4000/narratologie.10613

Ce document a été généré automatiquement le 6 septembre 2020

Article L.111-1 du Code de la propriété intellectuelle. 


\title{
Écosystème médiatique et circulation de la fiction : Dragon Ball, Pokémon, Sword Art Online
}

\author{
Bounthavy Suvilay
}

1 Pour décrire les phénomènes d'adaptations transmédiatiques contemporains, la majorité des recherches s'appuient sur des corpus occidentaux afin de montrer l'importance du personnage (Bertetti 2014, Aubrun et Lifschutz 2017) ou de l'univers de fiction (Klastrup et Tosca 2004, Johnson 2013) en tant qu'élément de cohérence liant les productions issues de différents secteurs. D'autres tentent d'élaborer un modèle dynamique où le processus de transformation donne lieu à des expressions spécifiques (Di Filippo 2017). De son côté, Marie-Laure Ryan critique le caractère excessivement théorique de la notion de transmedia storytelling de Henry Jenkins (2013), et elle souligne la différence majeure entre la structure décisionnelle top down qu'elle implique et la réalité des pratiques où les créations de franchises s'élaborant de manière bottom up (Ryan 2016). Des cas d'études japonais contemporains nous permettront de comprendre la variété des formes d'adaptations transmédiatiques et de montrer la manière dont le support initial et le contexte de production local influence les formats des déclinaisons.

2 Nous reprenons l'hypothèse évolutionniste de Linda Hutcheon développée dans $A$ Theory of Adaptation: "stories adapt just as they are adapted» (Hutcheon $2013: 31$ ). Elle utilise une analogie biologique pour exprimer le fait que les fictions narratives évoluent par adaptation au fil du temps, qu'elles migrent vers des environnements aux conditions favorables. Au Japon, l'écosystème médiatique est particulièrement propice aux adaptations et continuations de toutes sortes, mais il est nécessaire de délaisser la position anhistorique pour s'intéresser à l'évolution des industries culturelles au niveau local, ce qui permet de voir de quelle manière la fiction s'adapte à un milieu changeant.

Dans une perspective diachronique, nous proposons de montrer l'impact des rapports de forces entre les différents secteurs japonais de production culturelle sur les fictions locales. Après avoir rappelé le fonctionnement du media mix, circulation 
transmédiatique majoritairement centrée sur des déclinaisons d'un même récit, nous tenterons de montrer comment le jeu vidéo bouleverse cette configuration en transposant sur différents média un même univers fictionnel homogène géré de manière top down. Les problèmes contemporains liés à l'émergence de nouvelles formes de diffusion entraînent des formes d'adaptations sérielles inédites où les supports sont interrogés dans leur fonction de médiation.

\section{Adapter un récit : le manga comme source}

La prépondérance économique de l'imprimé au Japon s'explique par le fait que beaucoup de média audiovisuels sont dès leur origine liés aux maisons d'édition. Contrairement à la France où la télévision est longtemps restée un monopole d'État et un élément perçu comme dangereux par les concurrents historiques que sont les industries du papier (livre et presse) et la radio, au Japon les chaînes appartiennent généralement à des sociétés de presse ${ }^{1}$ : celles-ci se sont diversifiées en s'appropriant les nouveautés technologiques (Kinsella $2000: 6$ ). L'absence de concurrence entre les supports audiovisuels et imprimés a favorisé les synergies afin de toucher toutes les franges du public.

\section{Prévalence des industries du papier}

5 Le modèle du processus d'adaptation du manga (bande dessinée japonaise) en anime $e^{2}$ est relativement ancien et n'est pas propre au Japon. Toutefois, une convergence économique inédite s'est instaurée dans l'archipel. Initié en 1963 par l'adaptation par Osamu Tezuka du manga Tetsuwan Atom en série télévisée, le système du media mix utilise l'anime comme une forme de publicité pour recruter un nouveau lectorat (Steinberg 2012). Celui-ci paie pour avoir accès à la suite de l'intrigue en lisant la bande dessinée. Ce premier exemple a favorisé une convergence économique inédite des média. Tezuka a vendu à bas coût les épisodes à une chaîne, ce qui l'a contraint à faire appel à un sponsor pour compenser la perte financière. En contrepartie, celui-ci pouvait employer les visuels de l'anime afin de promouvoir ses produits (Steinberg 2012 : 37-86). De cette manière, le héros de la série devient omniprésent, représenté à la fois dans la presse, dans les livres, à la télévision, sur les affiches et sur les objets de consommation courants dans les supermarchés. La vente à bas coût des épisodes entraîne à la fois l'utilisation d'une animation limitée et la création d'une alliance multisectorielle entre la télévision, le livre et le sponsor qui utilise les visuels de la série pour promouvoir ses produits. Atom est ainsi présent sur de nombreux supports matériels, maximisant l'exposition médiatique du titre.

6 Dans cette configuration prépondérante dans l'industrie culturelle japonaise jusqu'au milieu des années 1990, l'œuvre source appartient à un auteur et son éditeur papier lui sert souvent d'agent en charge de former des partenariats qui permettent de produire les transpositions sur d'autres supports. Afin d'améliorer la rentabilisation des licences pour chaque média, le media mix fondé sur un manga impose une chronologie conçue pour optimiser la visibilité médiatique, tout en préservant la tension narrative sur tous les supports. La mise en place de cette stratégie doit sans doute plus aux opportunités et aux contraintes spécifiques à chaque média qu'à une volonté délibérée de gérer précisément la réception transmédiatique. Le circuit d'adaptation débute par le manga, 
passe par la série télévisée et les autres supports audiovisuels avant de finir par le jeu vidéo.

7 Autrement dit, la fiction narrative élaborée sur le support le plus low tech et demandant le moins de personnel créatif est transformée de support en support pour aboutir dans le secteur du jeu vidéo, plus récent et plus high tech. Le récit est donc initialement conçu dans un secteur dont le temps de production est particulièrement court (une semaine pour un chapitre) avant d'être décliné dans celui dont le rythme de production est le plus lent (plusieurs semaines dans le cas de l'anime et plusieurs mois pour le jeu vidéo) ${ }^{3}$. Ainsi, toutes les semaines, le public peut lire le nouveau chapitre de Dragon Ball (1984-1995) par Akira Toriyama dans le magazine Shônen Jump ${ }^{4}$ avant de regarder quelques jours plus tard l'adaptation animée réalisée par Toei Animation (5 séries d'animation et 19 films), puis d'acheter le volume relié (toujours publié par Shueisha), les jeux vidéo (110 jeux entre 1986 et 2015) et autres produits dérivés édités par Namco Bandai. La diffusion périodique du récit entraîne une ritualisation des consommations papier et audiovisuelle, qui se complètent.

Le passage d'un support à un autre implique également des modifications profondes du récit. Comme le souligne Philippe Marion, «Toute forme de représentation implique une négociation, voire un corps à corps, avec la force de résistance propre au système médiatique choisi » (Marion 1997 : 78). Nous ajouterons que les conditions de diffusion et la portée de la circulation modifient l'œuvre autant que les affordances techniques de chaque support.

\section{Récits réticulaires}

9 L'adaptation du manga vers la série est relativement fidèle au récit initial d'Akira Toriyama, alors que les films sont plus libres (contenus inédits issus du même univers diégétique ou non). Dans le cas de l'anime, la transposition impliquant une traduction intersémiotique selon un planning particulier, les récits ne sont jamais identiques et forment des univers diégétiques parallèles divergents. Afin de maintenir la chronologie des média et le décalage de près de deux mois entre chapitre de manga et épisode télévisé correspondant, Toei produit soit des expansions de segments narratifs apparaissant dans le manga, soit de nouvelles portions de récit.

Dans le premier cas, il s'agit de modifications liées à la médiativité (Marion 1997)5 à la traduction intersémiotique d'un support à un autre. En l'occurrence il s'agit du passage d'un support hétérochrone (papier) à un média homochrone (audiovisuel) ${ }^{6}$, modifiant les rapports au temps réel de l'énonciation médiatique (Marion 1997). Dans le second cas, les scénaristes insèrent des « interpolations transfictionnelles » (Saint-Gelais 2011 : 84-92), des expansions narratives désignées par le terme filler ${ }^{7}$. En effet, comme le format d'épisode de 25 minutes est bien trop long pour une adaptation fidèle du récit issu du manga ${ }^{8}$, des séquences inédites sont insérées et les réalisateurs doivent faire en sorte que ces ajouts soient à la fois fidèles à l'esprit du manga et intéressants pour un spectateur ne le lisant pas. Pour le téléspectateur connaissant déjà le manga, ces fillers accréditent la possibilité d'aller au-delà du récit exposé dans la bande dessinée et donnent de la consistance à l'univers diégétique.

11 Le suspense généré à chaque épisode pousse le téléspectateur à lire le manga où le récit est plus avancé afin d'apaiser son besoin de connaître la suite des événements. En un sens, le manga et les produits dérivés possèdent une fonction supplétive par rapport à l' 
anime: ils permettent de combler l'attente du prochain épisode en proposant de réinterpréter les événements avec des jouets, de lire immédiatement la suite et de mettre fin aux interrogations soulevées par le cliffhanger. Ainsi, les jouets et les figurines Dragon Ball sont des supports du mimicry, l'une des modalités du jeu selon Caillois (1992). Mais ces imitations enfantines ne prennent pas appui sur les comportements de personnages réels : elles reprennent les actions des personnages du dessin animé.

12 Autrement dit, au niveau de la réception, la série télévisée diffusée auprès d'une audience immense sert de réclame pour les média payants (manga, jouets et jeux vidéo). Elle est le principal levier de recrutement de lecteurs et de joueurs. Dans cette forme d'adaptation la narration et le marketing s'allient ainsi d'une manière particulièrement efficace.

13 Parallèlement à la série télévisée, les films produits chaque année proposent des variations inédites à partir de la même matrice narrative au lieu d'adapter le manga. La série animée peut ensuite agréger la trame narrative du manga et des longs métrages. Série, film et manga forment alors un réseau narratif où tous les éléments sont intriqués, mais qui ne forment pas un ensemble cohérent. La consistance progressive de l'univers fictionnel se fait par sédimentation des différents récits sans nécessairement gagner en cohérence interne.

Le manga Dragon Ball appartenant à un genre hybride (feuilleton reposant sur une dynamique sérielle), les lecteurs sont habitués au retour de certaines séquences (quête des boules magiques, entraînement et tournoi, transformation et montée en puissance). L'anime propose des variantes dans ses interpolations. Pour le téléspectateur n'ayant pas lu le manga, la tension narrative est produite par le suspense alors que pour les lecteurs regardant la série, elle nait de la curiosité : les incises transfictionnelles, qui proposent du contenu inédit, créent de nouvelles pistent d'intérêt, sans pour autant bouleverser le déroulement des événements déjà connus du récit tuteur.

15 En proposant des variantes à partir de la même matrice sérielle que le manga, les interpolations obligent l'auteur à innover pour ne pas répéter une situation déjà produite dans l'anime. Les scénaristes concurrencent ainsi le mangaka dans la déclinaison des péripéties possibles. Par ailleurs, Toriyama étant en charge des nouveaux personnages dans l'animation, il prend des décisions qui affectent l'univers fictif et le récit de son manga. Bardock, père de Goku, apparaît dans l'anime avant d'être représenté dans le manga, obligeant Toriyama à justifier après coup l'absence du personnage. Il n'y a donc pas une simple transposition d'un récit mais co-création alliant concurrence et émulation entre l'auteur de Shueisha et les créatifs de Toei qui élaborent en flux tendu et en parallèle les deux intrigues.

Si manga et anime bénéficient d'une même dynamique de création sérielle leur permettant de s'influencer mutuellement, le jeu vidéo est initialement le support le moins créatif en termes de narration. Dans la majorité des titres proposés, le joueur peut incarner le héros Son Goku ou ses compagnons afin de revivre les aventures déjà livrées en manga et à la télévision ${ }^{9}$ ou se battre contre les adversaires ${ }^{10}$. La déclinaison $\mathrm{du}$ récit en une forme vidéoludique se contente de reprendre et synthétiser les péripéties du manga et de l'anime sans apporter d'élément narratif nouveau. Le principal intérêt des jeux est de s'identifier au héros le temps d'une partie.

17 Jusque dans les années 1990, la matérialité du format et la périodicité des supports médiatiques imposaient au manga et à l'anime une logique de production sérielle en 
flux tendu et un circuit d'adaptation transmédiatique du récit par le biais duquel la fiction s'étend par interpolations et variations de manière non cohérente. Mais l'essor du jeu vidéo modifie la chaîne de déclinaison transmédiatique et le changement de support source transforme les formats de l'adaptation.

\section{Adapter un monde fictif : le jeu vidéo comme source}

Dans le cadre du media mix, plusieurs modèles de circulation de la fiction transmédiatique coexistent. Parallèlement au circuit d'adaptation partant du livre vers l'audiovisuel et le vidéoludique, d'autres possibilités ont été exploitées. À partir du milieu des années 1990, ces variantes du dispositif initial ont pris de l'ampleur, cette période correspondant à une perte d'influence des éditeurs papier. L'équilibre des forces entre secteurs économiques a récemment basculé en faveur du jeu vidéo qui impose ses prérogatives au cinéma ${ }^{11}$, mais aussi à la télévision et aux imprimés dans le cas du Japon.

\section{Prévalence du jeu vidéo}

19 Après la fin de Dragon Ball en 1995, le secteur du manga n'a cessé de décliner au Japon. Le Shōnen Jump qui était diffusé à 6,53 millions d'exemplaires par semaine en 1995 n'est plus diffusé qu'à 2,2 millions d'exemplaires en $2016^{12}$. Structurellement lié à la chute du taux de natalité du pays entraînant un rétrécissement du marché, ce déclin est accentué de manière conjoncturelle par l'attrait des nouveaux média.

20 Suite au lancement de la PlayStation en 1994, le jeu vidéo devient un produit grand public bouleversant l'équilibre des forces économiques et inaugurant un nouveau circuit d'adaptation où l'œuvre-source est un logiciel ludique. Les éditeurs ont alors commencé à adapter plus régulièrement des titres populaires issus du jeu vidéo comme Pokémon afin de recruter leur public. Le changement de médium-source manifeste le bouleversement de l'équilibre dans l'écosystème médiatique. Depuis la fin des années 1990, la multiplication des dispositifs de media mix non fondés sur un manga entraîne une évolution dans la création de ces fictions industrielles. Ce changement dans le support de l'œuvre-source rend manifeste le basculement hiérarchique entre les supports.

21 Affaiblie, l'industrie du papier s'appuie sur le système le plus fort pour tenter de récupérer son prestige économique et symbolique. Cette configuration économique se superpose à une situation de multiplication des plateformes, des média et des choix possibles pour les consommateurs. Si autrefois, les différents professionnels du secteur culturel pouvaient se coordonner pour organiser la rencontre de l'offre et de la demande, de telles stratégies de gestion du temps de réception sont dorénavant bien plus difficiles à mettre en place. Les sociétés de consommation actuelles sont confrontées à l'hyperchoix ${ }^{13}$, qui se caractérise par la possibilité d'accéder à un volume historiquement inédit de contenus sur une très grande diversité de supports ${ }^{14}$.

Gō Itō note le changement dans les modes de consommation et la chronologie des média, le public entrant dans la fiction par le jeu vidéo avant de lire des mangas bien plus tard (Itō 2005). L'émergence des Gangan comics souligne ce phénomène. Il s'agit de magazines de prépublication ciblant un public ado-adulte et proposant généralement des adaptations de jeux vidéo en bandes dessinées. Selon Itō, au lieu de montrer des 
personnages ou kyarakutaa (キャラクター) ${ }^{15}$, le manga met désormais en scène des kyara (キャラ), personnages tronqués de leur histoire, proto-personnages source de projection identificatoire du consommateur. Cette analyse correspond à la vision Hiroki Azuma qui, dans son essai de 2001, décrit la troisième phase de la culture otaku comme celle de la disparition des grands récits et l'avènement postmoderne d'une consommation de personnages présentant des «éléments d'attraction» (Azuma 2009). L'hégémonie de l'objet ou de l'image impliquerait que la collection devienne le noyau des pratiques culturelles.

La convergence économique ne s'établit plus autour d'un récit à adapter sur divers supports. D'ailleurs, au niveau de la production, l'intrigue a moins d'importance que la définition des caractéristiques des protagonistes. C'est ce que soulignent les recherches ethnographiques de Ian Condry dans le domaine de la création de série télévisée : "Cette combinaison de personnages (kyarakutā), de lieux (settei) et de mondes (sekaidan) a généralement précédé l'écriture de l'histoire en soi et peut donc être considérée comme une plate-forme de créativité pour l'anime $»^{16}$ (Condry $2013: 56$ ).

\section{Revisiter le monde fictif}

24 Le titre archétypal de cette phase d'évolution des industries culturelles japonaises est Pokémon. Lorsque Nintendo publie ce titre en 1996 sur Game Boy, console vieillissante aux graphismes en bichromie, personne ne prévoit son succès qui accroît les ventes de la console. L'éditeur Shogakukan acquiert les droits pour élaborer un manga publié dans le magazine pour enfant Koro Koro et une série animée est lancée en avril 1997 sur TV Tokyo. Ces adaptations renforcent la visibilité du jeu et se déclinent ensuite sur tous les supports. Depuis 1996, la licence Pokémon a engendré 212 jeux vidéo, une série télévisée toujours en cours (947 épisodes), 19 longs métrages, des jeux de cartes à collectionner, plusieurs séries de mangas, des jouets et figurines en tous genres. Si le début du processus d'adaptation reste bottom up, l'exportation et la suite de l'exploitation suivent une stratégie top down où toutes les actions sont coordonnées par la Pokémon Company, société regroupant Nintendo et le studio de création Game Freaks.

Selon Hutcheon, au lieu de proposer des adaptations de récits, le jeu vidéo favorise les traductions intersémiotiques d'un univers fictif, ce qu'elle désigne par le terme " heterocosm » (littéralement " autre cosmos ») (Hutcheon 2013 : XXIV). Dans le media mix ayant pour source un jeu, le monde fictif devient le décor de multiples intrigues se matérialisant sur d'autres formats (papier, audiovisuel...). Les jeux Pokémon de la série initiale proposent au joueur de parcourir le monde afin de collecter tous les monstres possibles et de devenir le meilleur dresseur de son territoire. Cette version moderne du récit initiatique permet au joueur d'incarner une histoire inédite, actualisant un « récit émergent ${ }^{17} »$.

Dans un jeu vidéo, il n'y a pas une trame narrative déjà tracée que le joueur recrée, mais une multitude de possibilités qu'il choisit d'activer ou non. La série télévisée comme les mangas proposent en quelque sorte une expression possible de ces récits émergents. Les spectateurs/lecteurs familiers du jeu peuvent reconnaître des mécaniques ludiques (phase de capture, phase de combat), des objets (Pokédex, Poké Ball), des personnages et des lieux, ce qui contribue à donner de la cohérence à 
l'univers fictif tout en assurant son développement puisque les récits papier ou audiovisuel ajoutent des informations sur l'histoire de ce monde imaginaire.

L'adaptation passe par la remédiation du jeu vidéo sur des supports antérieurs (manga et anime). Cette forme d'inclusion d'une technologie nouvelle dans un support plus ancien pourrait sembler aller à contre-courant du sens de l'histoire tel que semblent le définir Bolter et Grusin (1999), lorsqu'ils décrivent la remédiation comme la représentation d'un ancien média dans un nouveau ${ }^{18}$. Dans le media mix, elle se justifie par l'importance de la couverture médiatique qu'elle procure, qui permet de recruter des joueurs potentiels chez les lecteurs/spectateurs. Quant à ceux qui n'ont pas joué au jeu Pokémon, les adaptations permettent de rendre familier le gameplay et l'univers, ce qui facilite la prise en main du jeu, manga et anime constituant à la fois des tutoriels efficaces pour les futurs joueurs et une vitrine médiatique renvoyant à la source vidéoludique.

Par ailleurs, en dehors de la série principale de jeux vidéo, Pokémon propose aux joueurs de revisiter le même monde en adoptant d'autres points de vue. Au lieu d'incarner un dresseur, il est possible d'être un Ranger (humain empruntant les pouvoirs d'un Pokémon $)^{19}$ ou de devenir un Pokémon ${ }^{20}$. Par ailleurs, les titres offrent un large panel de gameplay. Dans Hey You, Pikachu! (1998), le joueur interagit avec un Pikachu et tente de le domestiquer en lui parlant. Pokémon Dash est un jeu de course où le joueur incarne un Pikachu tandis que dans Pokémon Snap, le joueur incarne un humain dont la mission est de photographier différents monstres dans une forme de safari.

Tandis que la licence Dragon Ball proposait la même histoire avec des variantes sur différents supports, Pokémon est un monde en constante croissance tant au niveau des récits qu'à celui des éléments qui le composent (chaque jeu étant l'occasion d'introduire de nouveaux monstres à attraper et des mécaniques de jeu nouvelles). Plus encore, alors que Dragon Ball imposait son rythme de lecture/visionnage au public à travers la découverte ritualisée d'un récit par une communauté d'interprétation recevant la fiction dans une même temporalité, Pokémon permet au joueur de s'affranchir de cette forme de rendez-vous. Il est toujours possible de rejoindre la communauté dans la vision simultanée d'un même épisode télévisé mais les joueurs peuvent échanger des monstres avec leur console portable quelle que soit l'avancée de leur expérience de jeu. Ce découplage entre temps de réception imposé du récit et formation d'un public partageant les mêmes références diégétiques change les formes d'appropriation de la fiction.

30 Les déclinaisons multisectorielles ne popularisent donc pas une trame narrative (cas Dragon Ball), mais un hétérocosme et ses règles. Chaque récit étant une suite possible d'événements en jeu, le consommateur peut aborder le monde par n'importe quelle entrée médiatique. La cohérence du monde fictif étant essentielle à la vraisemblance des différents récits, un lexique spécifique a été créé et des termes sont devenus des marques déposées (Pokémon et tous les noms de Pokémon). Lorsqu'une société souhaite traduire un récit de l'univers Pokémon (manga, anime, jeux, autres), elle reçoit d'ailleurs une bible comportant tous les noms traduits dans toutes les langues disponibles.

31 Dans le media mix japonais, cette nouvelle configuration de l'écosystème médiatique accroît les versions contrefictionnelles d'événements se déroulant dans un même univers ou, si l'on préfère, la prolifération d'univers parallèles incompatibles. En un sens, cette phase se rapproche du franchising défini par Johnson (2013): l'univers 
fictionnel étant partagé par des ayants droit multiples et vendu à d'autres sociétés, la cohérence globale est plus difficile à mettre en place.

\section{Adapter un concept ?}

Dans la troisième phase du développement des industries de contenus, la crise ne touche plus seulement le domaine du papier mais aussi celle du jeu vidéo forçant les sociétés à se restructurer et fusionner pour survivre ${ }^{21}$. SEGA arrête le développement de console pour devenir un simple éditeur à partir de 2001. Nintendo peine avec sa GameCube (2001) face à la Playstation 2 (2000) et perd ensuite une partie des core gamers avec la Wii et la WiiU qui ciblent le grand public.

\section{Crise généralisée}

Du côté de l'industrie du papier, la nécessité de l'export et de la prise en compte des publics étrangers dès la conception est d'autant plus pressante que le marché domestique ne cesse de se réduire ${ }^{22}$. Les sociétés d'édition ne parviennent ni à maintenir les ventes d'imprimés ni à développer suffisamment le marché des ventes numériques pour compenser les déficits ${ }^{23}$. Le premier éditeur de bandes dessinées au monde, Shueisha, annonce de son côté 4,18 millions de yens de perte en 2009. La situation ne s'améliore pas puisque l'Association japonaise des éditeurs de magazines (JMPA) indiquait que le tirage moyen de Shōnen Jump pour la période de janvier à mars 2017 était de 1915000 exemplaires, ce qui fait passer les ventes en dessous de la barre symbolique des deux millions, seuil qui avait été conservé jusque-là. Pour la $15^{\mathrm{e}}$ année consécutive, les ventes sont en chute et le marché du manga imprimé représente aujourd'hui la moitié de ce qu'il était au milieu des années $1990^{24}$.

Dans le secteur de l'audiovisuel, la situation n'est pas meilleure. Après l'explosion de la bulle économique du secteur de l'animation, les studios de production ont vu leurs conditions de travail et leur situation financière se dégrader pour aboutir à une série de faillites et de rachats en $2010^{25}$. Ceux qui restent survivent difficilement par la vente à des prix très élevés de Blu-Ray à une niche d'amateurs. Depuis la crise économique mondiale de 2008, les agences de communication et les chaînes télévisées faisant partie des comités de production des anime sont plus frileuses à l'idée d'investir dans des projets innovants dont le succès n'est pas garanti. Ce climat favorise les suites et les reboot de séries ${ }^{26}$. Il oblige les différents acteurs économiques à renforcer leur collaboration en vue de promouvoir une même licence. Outre la situation financière inquiétante, certains acteurs déplorent une perte de créativité27.

Pour expliquer cette crise des industries de contenus, certains évoquent l'essor du numérique et l'obsolescence du modèle économique du livre reposant sur l'achat d'un bien par un consommateur. Dans le domaine du jeu vidéo, le modèle du Free to Play basé sur un jeu gratuit avec des micro transactions occasionnelles s'impose face à celui de l'achat d'un logiciel. De même dans le domaine de la musique et celui de l'audiovisuel, le modèle de l'abonnement pour utiliser un bien tend à supplanter le modèle de la vente d'un produit (avec possession physique d'un objet ou d'un fichier).

Quelles qu'en soient les raisons, le déclin du livre et du jeu vidéo n'est pas spécifique au Japon. Mais il touche d'autant plus durement ce pays qu'il a été un leader dans ces secteurs. Au niveau de la création, les éditeurs ont de plus en plus recours à des 
amateurs qui se sont rendus célèbres sur internet et sont parvenus à se construire une communauté. Avant d'être publié en volume papier chez Shueisha depuis 2012, le bestseller One Punch Man était initialement un manga amateur élaboré par ONE et publié sur son site personnel depuis $2009^{28}$. L'éditeur recrute également au niveau international en organisant des concours de manga ${ }^{29}$.

$\mathrm{Au}$ niveau de la production, le marasme économique entraîne des modifications profondes dans les circuits d'adaptation et la chronologie des média. Au lieu de proposer une création concomitante en flux tendu du manga et de l'anime permettant la fidélisation d'un public autour d'un rendez-vous hebdomadaire, les séries d'animation sont écourtées et sortent plus tardivement ${ }^{30}$. La fragilisation du secteur de l'animation entraîne une réduction du nombre d'épisodes produits par série et un décalage plus grand entre l'avancée du récit initial (quel que soit le support source) et celle de l'anime.

Si l'on reprend le cas du media mix lié aux licences publiées par Shueisha, on remarque que beaucoup de séries ne font plus qu'une douzaine d'épisodes et qu'il faut attendre en moyenne un an ou deux entre chaque saison ${ }^{31}$. Ce nouveau modèle de production de l' anime contraste fortement avec le modèle précédent qui avait favorisé la pérennisation de Dragon Ball et One Piece grâce à la fidélisation d'un public hebdomadaire.

Contrairement à ce qui se produisait durant la première période, le format manga n'est plus le lieu où se développe de manière privilégiée un récit source qui est ensuite adapté et modifié dans d'autres secteurs. Il n'est plus l'un des terminus ad quem comme dans la deuxième phase, mais il devient l'un des possibles dans une généralisation des adaptations transmédiatiques.

\section{Jouer avec la frontière IG et IRL}

Dans cette troisième phase, l'un des cas les plus intéressants est Sword Art Online (SAO), light novel de Reki Kawahara ${ }^{32}$. Écrit en 2002 pour un concours de romans ayant pour thème le jeu vidéo, le texte a été publié sur internet sous un pseudonyme avant d'être édité en 2009 par ASCII Media Works, filiale de l'éditeur Kadokawa. Outre les séries de romans paraissant chez l'éditeur officiel, Kawahara publie également des dôjinshi, c'està-dire des œuvres à compte d'auteur distribuées sur le circuit des amateurs. Le roman est adapté en plusieurs séries de mangas dès 2010. Une première série animée voit le jour en 2012, suivie par un film (2017) et des jeux vidéo à partir de 2013. Le circuit d'adaptation ayant pour source un texte en ligne d'un auteur amateur dont le thème est un jeu culmine avec des jeux qui miment le $\mathrm{MMO}^{33}$ fictif sans en être au niveau du gameplay. Le décalage temporel entre les adaptations transmédiatiques contraste avec la mise en place de la licence hack qui avait déjà pour thème des joueurs de MMO prisonniers du monde virtuel et qui a décliné l'univers fictif sur tous les supports en un année $^{34}$. Le succès de l'évolution bottom up de $S A O$ contraste avec la stratégie top down de .hack, signe de la frilosité des différents acteurs économiques qui préfèrent attendre l'installation d'une communauté avant de se lancer dans des investissements coûteux (séries d'animation, jeux).

La licence $S A O$ relate les aventures de Kirito, joueur prisonnier d'un VRMMORPG ${ }^{35}$ avec 10000 autres personnes. S'il perd en jeu (IG ou In Game), il meurt dans le monde réel (IRL ou In Real Life) et s'il est déconnecté du jeu, il décède. Le seul moyen de revenir IRL est de parvenir à la fin du jeu après avoir vaincu les boss des 100 niveaux. Mais après 
être sorti vivant du jeu "Sword Art Online" se déroulant dans le monde fictif d'Aincrad, Kirito choisit d'aller dans ALfheim Online, autre VRMMORPG pour retrouver et sauver une joueuse qu'il avait rencontrée sur Aincrad. Par la suite, il joue à d'autres VRMMO où des incidents en jeu se répercutent dans le monde réel.

Malgré le titre, le héros passe relativement peu de temps dans le jeu SAO. Le roman, au lieu d'actualiser l'un des parcours possibles du joueur, met en scène un héros à travers plusieurs jeux imaginaires, déclinant ainsi le même concept de la frontière poreuse entre réalité et fiction. Récit d'anticipation dans le sens où l'auteur met en scène le futur proche du développement de la VR, SAO est généralement apprécié par les joueurs de MMO qui y retrouvent des thèmes, des mécaniques de jeu (guilde, arène de PVP) et des comportements de joueurs réels (Killer Player, personnes préférant vivre IG que IRL). Grâce à la remédiation de MMO dans les supports papiers puis audiovisuels, l'auteur n'a pas besoin de mettre des copyrights sur les mots comme dans le cas de Pokémon puisque ceux-ci appartiennent déjà au jargon des joueurs et permettent de faciliter l'identification.

En diégétisant les dangers d'un effacement entre IG et IRL, SAO ne vise pas à dénoncer les dangers du jeu vidéo mais prend acte de l'importance de ce loisir dans le monde contemporain et en joue dans une esthétique de la variation pour diversifier les univers fictifs seconds (les différents VRMMO auxquels jouent les personnages) tout en restant dans la même diégèse. Le roman interroge en réalité le support jeu vidéo, qui n'est plus un simple intermédiaire entre l'expérience du joueur en jeu et sa vie réelle.

Les transpositions de SAO sur d'autres supports ne constituent pas des adaptations de l'univers fictif mais des versions différentes de la même intrigue, voire des « variantes contrefictionnelles » (Saint-Gelais 2011 : 162) comme dans le cas des jeux vidéo. Dans la série des action-RPG adaptés de SAO, Kirito n'est pas sorti du VRMMO au niveau 75 (version du roman, mangas et anime) et doit vaincre les boss des 25 derniers niveaux. Autrement dit, si SAO comprend des remédiations du jeu vidéo et utilise le monde IG comme diégèse au sein de la diégèse, le support vidéoludique n'est pas un MMORPG, signe que le support d'origine de la fiction influe sur la forme de l'adaptation. Chaque série de mangas correspond aux aventures de Kirito dans un VRMMO et une quête différente, matérialisant ainsi la sérialité au cœur du roman répondant à l'injonction contradictoire de la répétition et de l'originalité.

Contrairement à ce qu'Azuma analyse à partir de la situation de l'écosystème médiatique au tournant des années 2000, il n'y a pas eu de disparition du récit mais celui-ci prolifère de manière différente. Au lieu de chercher la fidélité à une intrigue source (Dragon Ball à ses débuts) ou l'homogénéité d'un monde unique à travers ses actualisations (Pokémon), il faudrait s'intéresser à la déclinaison multisectorielle de concept, de propriété intellectuelle non industrielle.

Dans les processus de développement récents de Dragon Ball, la fiction n'a plus pour origine un manga mais des séries animées et vidéoludiques. Si les supports audiovisuels proposent des sequels, les jeux vidéo proposent des continuations métaleptiques où le joueur doit corriger une version contrefictionnelle du récit tel qu'il apparaît dans le manga et l'anime, la diégèse initiale étant annexée dans une fiction englobante située à un autre niveau. Les quêtes du MMO Dragon Ball Online et le récit de DB Xenoverse s'apparentent donc à la «capture transfictionnelle» (Saint-Gelais 2011: 234) où la fiction narrative dérivée inclut la diégèse initiale en tant qu'élément de l'histoire ${ }^{36}$. Dans le cas de Pokémon, l'« émancipation transfictionnelle du personnage » (Saint- 
Gelais 2011 : 373) permet également l'adaptation d'un concept. C'est le cas de Pikachu dans le jeu 3DS Meitantei Pikachu: Shin Konbi Tanjyou (2016). À la place de la collaboration entre un dresseur (le joueur) et un Pokémon lors de combat, le jeu d'aventure propose d'incarner le duo collaboratif de Sherlock Holmes (Pikachu) et Watson (le joueur). Quant au jeu en réalité augmentée Pokémon Go sorti peu après, il permet de superposer des éléments IG à un espace réel, le joueur n'ayant plus besoin d'incarner un avatar mais agissant directement en jeu.

Dans les trois cas, la fiction devient un élément ludique où la diégèse propose une diégèse seconde à modifier $(D B)$, une collusion dangereuse entre IG et IRL qu'il convient de séparer $(S A O)$, une variation hybridant une autre diégèse ou superposant espace virtuel et réel (Pokémon). Plus encore, les trois licences interrogent le support comme forme de médiation, comme si le numérique, en rendant les matériaux physiques obsolètes, pouvait abolir la frontière entre fait et fiction (Lavocat 2016). Cette complexification des narrations n'efface pas les régimes de production antérieurs, puisqu'en synchronie les trois formes d'adaptation coexistent, Shueisha continuant notamment de pourvoir le media mix en mangas adaptés ensuite selon le même circuit que $D B$. Mais elle témoigne de la prégnance du média vidéoludique et de la permanence du récit comme forme de structuration et d'entrée dans l'univers diégétique.

\section{Conclusion}

Nous avons postulé que les conditions socio-économiques de la production influaient sur les logiques d'adaptation, les rapports de force symboliques et économiques entre média ayant une incidence directe sur le format dans lequel se déploie le récit. Il s'agit de comprendre comment les supports et les contraintes de diffusion sont reformulés en termes créatifs. Après une première phase où l'industrie du papier était dans une position de force, la deuxième période voit l'émergence du jeu vidéo, support qui incarne la modernité et où s'élaborent des formes d'adaptation d'un monde fictif et non d'un récit. Plus récemment, l'essor du numérique bouleverse les configurations antérieures sans proposer de nouveau modèle économique viable. La crise des supports se traduit de manière diégétique dans les diverses remédiations et jeux sur la porosité entre fiction et monde réel qui mime la perméabilité entre concepteur et audience. L'œuvre source n'est plus produite au sein de l'écosystème médiatique officiel mais dans les circuits de création et de distribution des amateurs avant d'être réintégrée dans un cycle d'adaptation multisectorielle de l'industrie culturelle. Les tensions entre les circuits de consommation pensés par les acteurs du marché et les pratiques du public montrent que le braconnage productif des amateurs gêne le développement du secteur légal mais constitue aussi un réservoir de possible renouvellement des fictions.

La prise en compte des spécificités du contexte japonais permet de repenser des concepts tels que le transmedia storytelling (Jenkins 2013) en soulignant l'importance du support sur l'expression du récit et en réintroduisant le facteur temps (chronologie des média dans les processus d'adaptation, évolution des pratiques selon les rapports de force des secteurs économiques). Au lieu d'établir une simple nomenclature descriptive anhistorique, il s'agit d'étendre l'histoire littéraire à l'ensemble des domaines de fictions narratives contemporaines en prenant en compte les matériaux médiatiques. 


\section{BIBLIOGRAPHIE}

Anderson, Chris (2009), La longue traîne - La nouvelle économie est là !, Paris, Pearson.

Aubrun, Frédéric, Lifschutz, Vladmir (2017), « Le traitement médiagénique de Batman. Des franchises au transmedia storytelling ", Communication, en ligne, vol. 34/2, consulté le 4 décembre 2019. URL : http://journals.openedition.org/communication/7376

Azuma, Hiroki (2009), Otaku : Japan's database animals, Minneapolis, University of Minnesota Press. Bertetti, Paolo (2014), « Toward a Typology of Transmedia Characters », International Journal of Communication, $\mathrm{n}^{\circ} 8$, p. 2344-2361.

Blanchet, Alexis (2010), Des pixels à Hollywood. Cinéma et jeu vidéo, une histoire économique et culturelle, Châtillon, Pix'n Love.

Bolter, Jay \& Richard Grusin (1999), Remediation : Understanding new media, Cambridge, MIT Press. Caillois, Roger (1992), Les Jeux et les hommes : Le masque et le vertige, Paris, Folio.

Condry, Ian (2013), The soul of anime : collaborative creativity and Japan's media success story, Durham, Duke University Press.

Dena, Christy (2009), Transmedia Practice : Theorising the Practice of Expressing a Fictional World across Distinct Media and Environments, thèse de philosophie sous la direction de Gerard Goggin, Université de Sydney.

Di Filippo, Laurent (2017), « Conan entre personnage transmédia, monde transmédial et réalisations locales ", Revue française des sciences de l'information et de la communication, en ligne, $\mathrm{n}^{\circ}$ 10, consulté le 4 décembre 2019. URL : https://journals.openedition.org/rfsic/2578

Hutcheon, Linda (2013), A Theory of Adaptation, $2^{\text {nd }}$ edition, London, Routledge.

Itō, Gō (2005), Tezuka izu deddo : hirakareta manga hyōgenron e, Tokyo, NTT Shuppan NTT.

Jenkins, Henry (2013), La Culture de la convergence. Des médias au transmédia, Paris, Armand Colin / INA, traduction française.

Johnson, Derek (2013), Media franchising: Creative license and collaboration in the culture industries, New York, NYU Press.

Juul, Jesper (2005), Half-Real : Video Games between Real Rules and Fictional Worlds, Cambridge, The MIT Press.

Kinsella, Sharon (2000), Adult Manga, Honolulu, University of Hawaii Press.

Klastrup, Lisbeth \& Susanna Tosca (2004), « Transmedial Worlds - Rethinking Cyberworld Design ", in Proceedings of the 2004 International Conference on Cyberworlds, M. Nakajima, Y. Hatori \& A . Sourin (dir.), Los Alamitos, CA, IEEE Computer Society, p. 409-416.

Larceneux, Fabrice, Sophie Rieunier, \& André Fady (2007), « Effet de l'hyperchoix sur le consommateur et effet modérateur de la marque : une application au cas de l'horlogerie bijouterie ", Recherche et Applications en Marketing, 22.4, p. 43-56.

Lavocat, Françoise (2016), Fait et fiction - Pour une frontière, Paris, Seuil.

Marion, Philippe (1997), « Narratologie médiatique et médiagénie des récits », Recherches en communication, $\mathrm{n}^{\circ} 7, \mathrm{p} .61-87$. 
Mick David Glen, Susan M. Broniarczyk \& Jonathan Haidt, « Choose, Choose, Choose, Choose, Choose, Choose, Choose : Emerging and Prospective Research on the Deleterious Effects of Living in Consumer Hyperchoice ", Journal of Business Ethics, 52, 2004, p. 207-211.

Ryan, Marie-Laure (2016), « Mondialité, médialité », in A. Besson, N. Prince \& L. Bazin (dir.) Mondes fictionnels, mondes numériques, mondes possibles, Rennes, PUR, p. 21-39.

Saint-Gelais, Richard (2011), Fictions transfuges, la transfictionnalité et ses enjeux, Paris, Seuil. Steinberg, Marc (2012), Anime's Media Mix, Minneapolis, University of Minnesota Press. Toriyama, Akira (1999), Le Dictionnaire de Dragon Ball, Grenoble, Glénat.

\section{NOTES}

1. Par exemple, le réseau de chaînes télévisées Nippon Television Network System (日本テレビ ネットワーク協議会) est contrôlé par la société ayant créé le premier quotidien en terme de ventes au Japon, le Yomiuri Shimbun (読売新聞) fondé en 1874, diffusion de 10 million d'exemplaires en 2010). Le deuxième quotidien, Asahi Shimbun (朝日新聞depuis 1879), appartient au groupe qui a développé TV Asahi Channel et FM OSAKA (radio).

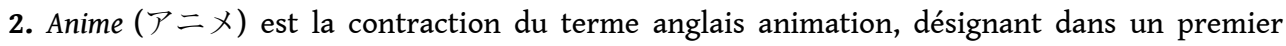
temps tous types de production de dessin animé, mais le plus souvent utilisée de nos jours pour l'animation pour la télévision.

3. Pour un exemple de temps de production et de chronologie des média, voir Dena (2009: 81$)$.

4. Cet hebdomadaire avait une diffusion de 6,53 millions d'exemplaires à son pic d'audience en 1995.

5. Néologisme calqué sur le terme narrativité, la médiativité correspond à la singularité différentielle d'un média, c'est-à-dire «tous les paramètres qui définissent le potentiel expressif et communicationnel » d'un média (Marion $1997: 80$ ).

6. Pour Marion (1997), dans un «média hétérochrone» le temps de réception n'est pas programmé par le support, tandis que dans un support «homochrone » le temps de réception dans l'énonciation de ses messages.

7. De l'anglais to fill « remplir ». Les fillers désignent généralement des épisodes n'étant pas adaptés du récit mais reprenant les éléments de l'univers avec des événements inédits afin de temporiser la suite de l'adaptation.

8. C'est ce que rappelle Akira Toriyama dans une de ces BD explicatives en marge de Dragon Ball (Toriyama 1999 : 157).

9. Cas des jeux d'action-aventures comme Dragon Ball: Le Secret du Dragon (1986).

10. Cas des jeux de combat comme la série des Dragon Ball Z Budokai (2002-2007).

11. Cette constatation vaut pour la plupart des pays occidentaux dont la France. «Le cinéma a de plus en plus un statut de produit d'appel, et la salle une vocation de vitrine, de système promotionnel pour une valorisation marchande qui s'effectue pour l'essentiel sur d'autres supports » (Blanchet $2010: 274)$.

12. Entre 1994 et 2004 les ventes de manga ont baissé de $20 \%$ mais la bande dessinée représentait encore $37 \%$ des publications vendues et $23 \%$ du chiffre d'affaires dans le secteur de l'imprimé. Voir le rapport du JETRO de 2005.

13. Le contexte d'hyperchoix dans à une société de consommation a surtout été décrit dans le domaine du marketing comme une surabondance de possibilités rendant la prise de décision difficile (Mick, Broniarczyk, Haidt 2004, Larceneux, Rieunier, Fady 2007). 
14. Chris Anderson, rédacteur en chef du magazine Wired a longuement développé sa théorie du paradis des consommateurs dans un essai portant sur le marketing à l'ère numérique (Anderson 2009).

15. Transcription phonétique de l'anglais Character.

16. Ma traduction de: "This combinaison of characters (kyarakutā), premises (settei), and worlds (sekaidan) generally came before the writing of the story per se and thus can be considered a platform of anime creativity. » (Condry $2013: 56$ ).

17. Le récit émergent tend à se confondre avec l'expérience du joueur : «Emergent narrative tends to be described very loosely as the player's experience of the game [...], or the stories that the players can tell about the game, or, perhaps, the stories that players can create using the game. » (Juul 2005).

18. La remédiation est définie comme la représentation d'un support dans un autre : «Again, we call the representation of one medium in another "remediation," and we will argue that remediation is a defining characteristic of the new digital media. What might seem at first to be an esoteric practice is so widespread that we can identify a spectrum of different ways in which digital media remediate their predecessors, a spectrum depending upon the degree of perceived competition or rivalry between the new media and the old. » (Bolter et Grusin 1999: 45).

19. Série des Pokémon Ranger depuis 2006 (3 titres).

20. Par exemple la série des Pokémon Donjon mystère à partir de 2005 (5 titres).

21. Fusion de Square et Enix en 2003 ; de Namco et Bandai en 2005.

22. En 2008, Kodansha qui est pourtant le numéro deux du marché du manga affiche 7,7 millions de yens de perte.

23. Même si elles représentaient 42,8 millions de yens en 2009 , les ventes de mangas numériques ne permettent pas d'enrayer les pertes. En 2016, les ventes de mangas ont totalisé 194,7 millions de yens (baisse de 7,4\% par rapport à l'année précédente), tandis que les ventes de magazines de mangas ont totalisé 101,6 millions de yens (baisse de 12,9\% par rapport à l'année dernière).

24. Animēshonbijinesu jānaru, http://animationbusiness.info/archives/2406 (consulté le 1 février 2019).

25. En 2010, Group TAC, MadHouse et Future Planet ont déclaré faillite.

26. Lancée à l'occasion des vingt ans de la licence, Pretty Guardian Sailor Moon Crystal (2014) est une nouvelle série animée plus proche du manga original. De même pour les vingt ans de Dragon Ball, Toei Animation a lancé une version en HD de la série Dragon Ball $Z$ sous le titre Kai (2005-2015). Ghost in the Shell 2.0 (2008) est un remake du film de 1995 et les quatre moyens métrages Ghost in the Shell: Arise (2013-2014) forment un prequel à celui-ci.

27. La mort de l'industrie du jeu était annoncée par Keiji Inafune (créateur de Mega Man) au salon Tokyo Game show en 2009, tandis que celle de l'animation japonaise était prédite comme prochaine par le réalisateur Hideaki Anno (créateur de Neon Genesis Evangelion) en 2015.

28. De même Tetsuya Tsutsui était un mangaka amateur, repéré et publié par un éditeur français (Ki-oon) avant d'être publié au Japon par Shueisha, signe que si autrefois les Japonais exportaient des objets culturels vers l'Occident, aujourd'hui les Européens peuvent choisir des œuvres qui leur correspondent et les mettre en lumière dans leur pays d'origine.

29. En décembre 2015, il a lancé le Jump Plus X Medibang Manga Contest qui a recueilli plus de 900 propositions en trois mois. Voir le site officiel du concours https://medibang.com/jumpplus/ (consulté le 1 février 2019).

30. Paraissant de façon hebdomadaire dans Shōnen Jump comme l'avait été Dragon Ball en son temps, le manga Haikyû ! n'est pas adapté en une série télévisée diffusée toutes les semaines mais en plusieurs saisons dont le nombre d'épisodes est variable : 25 épisodes pour la première et deuxième saison (2014 et 2015-2016), 10 seulement pour la saison 3 (2016). Il n'y a plus autant de séries avec une diffusion continue tout au long de l'année avec des épisodes comportant des fillers.

Cahiers de Narratologie, 37 | 2020 
31. C'est le cas de la première saison de Boku no Hīrō Academia, la saison 2 de Shokugeki no Soma et bien d'autres séries issues du Shōnen Jump.

32. Un light novel est un court roman illustré destiné à un public de lycéens et de jeunes adultes.

33. Un MMORPG (Massively Multiplayer Online Role-Playing Game) est un jeu de rôle vidéo en ligne permettant à un grand nombre de joueurs d'interagir simultanément dans un monde virtuel persistant.

34. Élaboré par la société de jeux CyberConnect2, le transmedia storytelling de SAO se compose d'une série télévisée (.hack//Sign, 2002) proposant le récit d'un joueur de MMORPG et posant l'univers fictif qui est exploité dans les quatre jeux vidéo (qui permettent d'incarner d'autres personnages prisonniers du même MMO, 2002-2005), d'un roman .hack//Another Birth (4 volumes réinterprétant les scénarios des jeux, 2004-2005), d'un manga .hack//Legend of the Twilight (suite de .hack//Sign parue entre 2002 et 2004) et de son adaptation animée (2003).

35. Dans la diégèse, il s'agit un MMORPG qui se joue avec un casque de réalité virtuelle (VR) et des commandes cérébrales, les trois éléments (MMORPG, VR et interface cérébrale) étant déjà disponibles actuellement séparément.

36. La diégèse initiale apparaît en jeu sous la forme d'un volumen, remédiation du support papier où s'est initialement développée la fiction.

\section{RÉSUMÉS}

Dans une perspective historique, l'article propose de montrer comment les supports médiatiques et les conditions socio-économiques de la production influent sur les logiques d'adaptation : de la simple transposition d'un récit aux interpolations transfictionnelles, de l'adaptation d'un univers diégétique à la remédiation. Les rapports de force symbolique et économique entre média ont une incidence directe sur le format dans lequel se déploie le récit.

Based on Japanese transmedial fictions, this paper shows how the material and the production condition influence the adaptation process and the reception by the audience. The media chronology and the order of adaptation change the narratives, and each new iteration creates a specific case. We intend to show how the symbolic and economic power struggles between the media have a direct impact on the format in which the story unfolds.

\section{INDEX}

Index chronologique : XXe siècle, XIXe siècle

Mots-clés : adaptation, manga, bande dessinée, jeu vidéo

Index géographique : Japon

\section{AUTEUR}

\section{BOUNTHAVY SUVILAY}

Après une thèse sous la direction de Marie-Ève Thérenty (RIRRA21, Montpellier 3) et de Matthieu Letourneux (PHisTeM-CSLF, Paris-Ouest), Bounthavy Suvilay s'intéresse aux adaptations 
transmédiatiques, leurs circulations globales et les phénomènes de (re)création et/ou resémantisation au niveau local (traduction, adaptation, réception distincte selon les publics). Bounthavy Suvilay, « Le "Cool Japan” made in France. Réappropriation du manga et de l'animation japonaise (1978-2018) », Ebisu en ligne, 56 | 2019, 71-100. https://

journals.openedition.org/ebisu/3666

Bounthavy Suvilay, «Adaptation transmédiatique : Le Tour du monde en série animée hispanojaponaise », in Maxime Prévost (dir.) Jules Verne et la culture médiatique. De la presse du XIXe siècle au steampunk, Québec, Presses de l'Université Laval, 2019, p. 169-192.

Bounthavy Suvilay, Édith Taddei, « Les mangas : Faire entrer les lectures privées à l'école et les constituer en objets littéraires ", Le Français aujourd'hui №207 (4/2019), pp. 81-91, Armand Colin. Disponible sur : https://www.revues.armand-colin.com/lettres-langues/francais-aujourdhui/ francais-aujourdhui-no207-42019/mangas-faire-entrer-lectures-privees-lecole-constituer-objetslitteraires 